\title{
Research and Design of Peanut Diseases Diagnosis and Prevention Expert System
}

\author{
Kun Zhang, Benjing Zhu, Fengzhen Liu, and Yongshan Wan* \\ College of Agronomy, State Key Laboratory of Crop Biology, \\ Shandong Agricultural University, Taian 271018, China \\ $\{$ kunzh, yswan\} @sdau.edu.cn
}

\begin{abstract}
Based on the analysis of characteristics and rules of the peanut diseases, this article sums up the table for peanut diseases diagnose, presents an expert system for peanut diagnose, information service. The architecture, functions, knowledge base and inference engine are designed. The system will greatly improve the prevention of peanut diseases.
\end{abstract}

Key words: Peanut diseases, Diagnose, Expert system.

\section{Introduction}

There are many types of peanut diseases, happen frequently, which seriously affected the yield and quality of peanut. The occurrence of disease is affected by many factors. It is complex, boundary fuzzy, uncertainty, poor controllability (Wan, 2003; Wang, 1999; Sun, 1998). Due to the shortage of basic technical personnel and lack of knowledge, when peanut diseases occurs, the correct diagnosis and proper treatment can not be given at the first time, which bring about a large area of peanut diseases and seriously impact on the yield and quality of peanut. Therefore, the scientific and timely for the diagnosis and treatment of the diseases of peanut would be improved through studying the method of diagnosis and treatment of diseases of peanut and setting up peanut diseases diagnosis and prevention expert system. It's especially good for the diagnosis and prevention of major peanut diseases, which would greatly enhance the level and efficiency of peanut diseases prevention and control.

There are lots of research and application for plant protection expert system abroad, and there are many successful cases. Such as PLANT/ds soybean disease diagnosis expert system developed by the University of Illinois is the earliest development for agricultural expert system in the world. The tea (Camellia Sinensis) pest expert system (TEAPEST) developed by Ghosh (2003) is object-oriented expert system based on rules. It can identify the most diseases of tea plant, and achieved very good results in practical application. Apple disease management decision-making expert system developed by S. Haley can help farmers by providing advice, how to improve the apple

\footnotetext{
* Corresponding author.
} 
production, and can give proper means to diagnosis after identify the species, and this system is suitable for the less experienced personnel.

In recent years, research and application of agricultural expert system in China developed quickly. Many agricultural expert system has been applied in practice and played an important role, and also has made great economic benefits and social benefits. Such as "corn diseases and insect pests prevention expert system" implemented by Jilin University and Jilin Academy of Agricultural Sciences, is one for the farmers based on uncertainty reasoning, multimedia and friendly interface technology (1999). Important vegetable pest diagnosis and consultation system developed by Zheng Yongli (2004) in Zhejiang Province is programmed by the object-oriented relational database Visual FoxPro 8.0, and it adopted the event-driven model such as properties, methods and events programming. While as for peanut, there is no expert system of peanut pest identification, which is systematic and helpful for users. Therefore, we would develop a peanut diseases diagnosis and prevention expert system.

\section{The System Development Tools}

The software is designed based on the operating system Windows XP, using VB6.0 as the expert system programming tool, Access 2003 as the background database, Dream-weaver as HTML browsing software, Photoshop6.0 to process the images.

\section{Structure and Function Design of the System}

\subsection{Structure of the System}

The system is developed using the modular design thought. It's composed of inference engine, database, knowledge base, system maintenance and user interface, etc. The core of the system is the knowledge base and inference engine. System has a modular structure, each module is independent, and the knowledge database and inference engine is separated, so it is easy to maintain and update.

\subsection{Function of the System}

In the principle of usability, the system realized the function of peanut disease diagnosis, disease information querying, scientific application of pesticides consultation and knowledge base maintenance, etc. Each functional module is described as follows:

\subsubsection{Disease Diagnosis}

Diagnose the diseases which have occurred in the current field, according to the characteristics of symptoms of disease the user supplied; then all the information of the diseases diagnosis will be given, including symptoms, etiology, pathogenesis, prevention measures and the picture for the characteristics. 


\subsubsection{Disease Information Querying}

The module provides advisory function for the user. It mainly provides disease symptoms, occurrence, control methods and other aspects of the information queried. There are five types of disease included, namely, fungal, bacterial diseases, viral diseases, nematodes disease, and noninfectious diseases. Users can query the information, learnt identify, improve the correct rate of the recognition only a simply click of the mouse.

\subsubsection{Advisory Scientific Medication}

Mainly to provide service of retrieval, query, consulting and learning about relevant information about commonly agricultural chemicals used in peanut. For example, characteristics of pesticide, the commodity name, English name, Chinese name, and control object toxicity, control method and characteristics of the function and so on.

\subsubsection{System Maintenance}

This part mainly used for maintenance of system database, knowledge base, picture library editing, updating, deleting, expansion and system program, etc.. Also it can detect the consistency and integrity of knowledge, to avoid redundant or the contradictions of old and new knowledge in the knowledge base, and make certain limits of user authority to ensure the normal operation of the system.

\section{$4 \quad$ Realization of System Functions}

\subsection{Establishment of Database}

The system contains disease database, pesticide database and disease diagnosis database. The structure and examples of the three databases are as follows (table 1., table2., table 3.).

Table 1. Structure and examples of diseases database

\begin{tabular}{|c|c|c|c|}
\hline Fields & Field type & Field length & Example \\
\hline Disease name & text & 14 & leaf spot of peanut \\
\hline alias & text & 30 & early leaf spot of peanut \\
\hline Symptoms & remarks & automatically & omit \\
\hline Pathogens & remarks & automatically & $\begin{array}{l}\text { Groundnut Cercospora, belonging to ascomycotina } \\
\text { fungi }\end{array}$ \\
\hline $\begin{array}{l}\text { Disseminated } \\
\text { cycle }\end{array}$ & remarks & automatically & omit \\
\hline Pathogenic factors & remarks & automatically & $\begin{array}{l}\text { The optimum temperature } 25-28 \cdot \text {, the onset heavy } \\
\text { in rainy and humid climate, }\end{array}$ \\
\hline $\begin{array}{l}\text { Prevention } \\
\text { method }\end{array}$ & remarks & automatically & Selection of resistant varieties, spraying pesticides \\
\hline Disease picture & OLE object & & omit \\
\hline
\end{tabular}


Table 2. Structure and examples of pesticide database

\begin{tabular}{llll}
\hline Fields & Field type & Field length & Example \\
\hline Chinese name & text & 10 & 多菌灵 \\
English name & text & 20 & carbendazim \\
Trade name & text & 30 & MBC \\
Categorical attribute & text & 20 & bactericide \\
Dosage & text & 100 & 25\%, 50\%carbendazol wettable powder \\
Control object & text & 200 & peanut seedling blight, stem rot, root rot, leaf spot, \\
Toxicity & remarks & automatically & Mouse acute oral LD50 8000-10000 mg / kg \\
Matter need attention & remarks & automatically & omit \\
\hline
\end{tabular}

Table 3. Structure and examples of diagnose of diseases database

\begin{tabular}{llll}
\hline Fields & Field type & Field length & Example \\
\hline Diseased location & text & 10 & leaf \\
Symptom 1 & text & 50 & spot round or irregular in shape \\
Symptom 2 & text & 50 & spot with halo around \\
Symptom 3 & text & 50 & Spots of dark brown, large, yellow halo, Grey \\
& & & Mildew account raw when wet \\
Disease name & text & 20 & cercospora brown spot of peanut \\
\hline
\end{tabular}

\subsection{Realization of Disease Diagnosis}

\subsubsection{Organization Structure of Knowledge}

Built diagnostic tree about disease (occurred in root, stem, leaf and plant) and characteristics of the damage, by collecting the characteristics of peanut diseases, summarizing these data and sorting out the peanut disease retrieval table, finally the making the retrieval table into diagnostic tree for computer program reasoning, as shown in figure 1. Each node in Fig. 1 corresponds to a group of knowledge. The method of diagnosis tree analysis will promote an unknown problem layer by layer, finally get the disease name. 


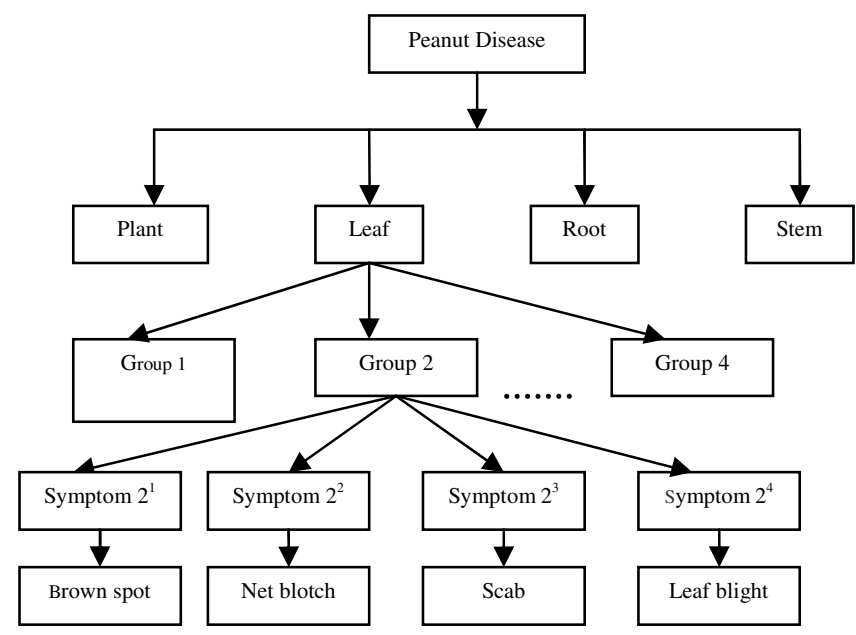

Fig. 1. The knowledge structure of peanut disease diagnose

\section{Note :}

Group 2 : Lesion round or irregular in shape.

Symptom 21 : Lesion tea brown or dark brown, with yellow halo, gray powder mildew in the wet spot surface

Symptom 22 : Stellate black spot at the beginning, chlorotic with halo, then reticulate brown spots, with small black protrusions.

Symptom 23 : The lesion sag middle, uplift at the edge, adaxially yellow-brown, caused perforation.

Symptom 24 : A dark brown irregular moire spot appears first in the tip and edge of the leaf, leaf rot off, when humidity is big, with white mycelium and sclerotium.

The first layer: Onset location, there are four parts, the whole plant, leaf, root and stem.

Second layer: Symptom group, which is the common features reorganized and extracted by a part of a disease experts and knowledge engineers.

Third layer: Characteristics of disease symptoms, is the further detailed description. This system is distinct uniformly by the symptoms of $1,2,3$. If give the conclusion directly by the symptoms of nodes, can get the final point, otherwise continue to establish the node, and so on, until all nodes come to the final node.

\subsubsection{Knowledge Representation}

The system adopts two levels including the rule frame and the body of the rule to describe the knowledge of disease diagnosis through the way of thinking and the hierarchical structure model that analysis of disease diagnosis. Rule frame reflects the 
logic relationship between the premise and conclusion factor, which is a reasoning framework; the rule body reflects the value knowledge between premise factors and conclusion factors, it can contain formulas, may also have a set of rules. Its general form is:

IF Ei(I) then A

The Ei $(1,2,3, \ldots \ldots, n)$, as the logic relationship of "and", Ei and A are collectively referred to "fact", "concept" or "factors" in "rule frame + rule body". Take the peanut leaf disease as an example:

RSn:IF lesion mildew layer

THEN Name of peanut diseases

RB: IF gray mold on lesion

THEN peanut brown spot

IF lesions with white mycelium and sclerotium

THEN peanut leaf blight

IF lesion dark brown, in concentric rings of spots, with dark brown mildew

THEN peanut brown spot

IF lesion brown by yellow, dark brown edge with a yellow halo, rupture, like anxiety, with small black spots

THEN peanut focal spot

Among them, RS represents the rule frame identifier, $\mathrm{n}$ is rule frame number, and is also the rule group number. RB is the rule body identifier, and the downward to the next rule frame identifier are all the rule body content in the rule group. In the system, the rule groups are mutual independence, the group number can be identified arbitrary, as long as not to repeat, that is place of the rule group is arbitrary. System will put similar knowledge in the same group rules, such as the diagnosis of diseases based on leaf symptoms, rule body expresses the solution process to the conclusion set organically and naturally, avoiding redundant and dispersion in writing rules, knowledge base is compressed greatly, and the speed of reasoning is accelerate greatly.

\subsubsection{Realization of Inference Engine}

The system adopts depth-first method to perform comprehensive reasoning, by analyzing the process of peanut disease diagnosis, summarizing the experts' advice and experience. The search tree for disease diagnosis established, and the search is from the top to down along the hierarchical structure of knowledge. The reasoning process of the system can be seen as a search process for the results along the search tree.

The basic idea of depth-first search is: start from the initial node $\mathrm{S}$, select a node to inspect among the child nodes, if not the target node, then select another node to inspect, search down so on and so on. When reach some child node, if the node is not the destination node also cannot continue to expand, and then select its sibling nodes to inspect. Take peanut leaf disease as an example: firstly, using reverse reasoning, to determine the position of peanut diseases is in peanut leaf, and then using the depth-first method forward reasoning, confirm the diagnosis group (i.e. initial node) for lesion plaque surface mildew layer, select a node in its child node to inspect, namely specific symptoms contained, if not the target node, then inspect in the next level of 
symptoms, search down until the diagnosis results are obtained. If the diagnostic results are inconsistent with the actual, this disease is not the target node and also cannot continue to expand, then reselect the symptoms to inspect, repeat the process, until the results are obtained.

\subsubsection{Main Program}

Programming method to realize the function of disease diagnosis is as follows.

Option Explicit

Public db1 As Database

Public str1 As String, str2 As String

Public rs1 As DAO.Recordset

Dim cn As Connection

Dim rs As Recordset

Private Sub Form_Load()

Text1.Text $=$ Date

Text2.Text $=$ "Taian in Shandong Province"

Text12.Text $=$ " Adult onset "

Text3.Text $=$ czqzd.Combo1.Text

Text 4 .Text $=$ czqzd.Combo2. Text

Text5.Text $=$ czqzd.Combo3.Text

Text6.Text = czqzd.Combo4.Text

Set db1 = OpenDatabase(App.Path \& "Idatabase.mdb")

'Match knowledge in rule base according to information the user selected

str1 = "select $*$ from bhzd where zz1 like "' + czqzd.Combo2.Text + "' and zz2 like"' + czqzd.Combo3.Text + "' and zz3 like "' + czqzd.Combo4.Text + "'"

Set rs1 $=$ db1.OpenRecordset(str1, dbOpenDynaset $)$

If rs1.BOF And rs1.EOF Then

MsgBox " There's no symptoms according to your diagnosis, please choose the symptoms correctly!", vbExclamation, "diagnosis Suggestion"

Else

Text7.Text $=$ rs1.Fields $(" b h m c ")$

rs1.close

db1.close

End If

Set adocon $=$ New Connection

adocon . CursorLocation $=$ adUseClient

adocon.open "Provider=Microsoft.Jet.OLEDB.4.0;Data Source=" \& App.Path $\&$ "\database.mdb"

Set rs = New Recordset

rs.open "binghai", adocon, adOpenDynamic, adLockOptimistic

rs.MoveFirst

On Error Resume Next

Do Until rs.EOF

If rs.Fields("Disease name ") = Text7.Text Then

Text8.Text = rs.Fields("alias ") 


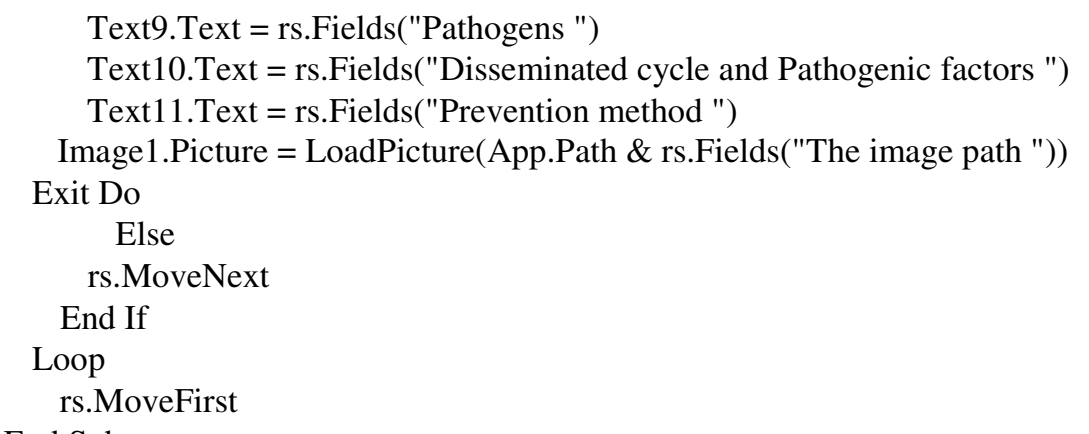

The operation interface of peanut diseases diagnosis is as follows.

Firstly, select disease symptoms according to the local actual situation (Fig.2). Then we get the diagnosis results after the "start diagnosis" button been clicked (Fig.3).

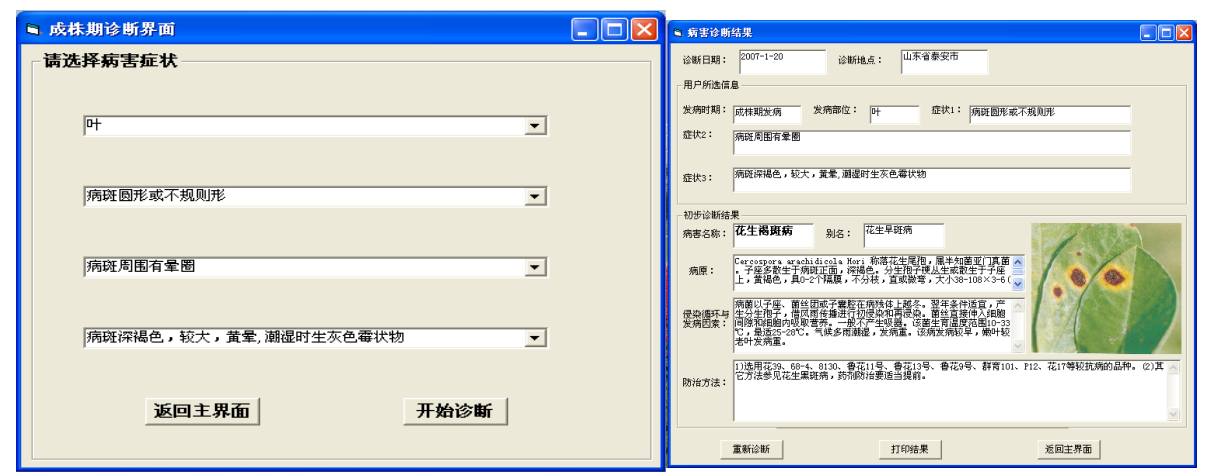

Fig. 2. Interface of the symptom diagnoses

Fig. 3. Result of the disease diagnose

\subsection{Realization of the Query Function}

The system will add the corresponding Chinese name exists in the peanut disease inquiries to the list of user interface box, gain the corresponding diseases of picture and text information, by selecting one of the Chinese name or calling the query function internal system by inputting known Chinese name. Retrieval function by English name or Chinese name is basically the same in design method. Take the disease Chinese name search as example; the programming method of realizing the retrieval functions is as follows:

\section{Private Sub cmdfind_Click ( )}

' 'Button after the user complete entering in the text box, to trigger the retrieval event, program starts'

Data1.RecordSource $=$ "select $*$ from" $\&$ binghai $\&$ "where disease name $=" \&$ "'" \& txtname.Text \& "'" 
' 'Find the corresponding Chinese name in the library'

If Data1.Recordset.recordcount $<=0$ Then

MsgBox "sorry, there is no information about what you find, make sure you typed it correctly"

Data1.RecordSource $=$ "select $*$ from" \& binghai

End If

"'There is not the corresponding Chinese name to find in the library, display a message' If Data1.Recordset.recordcount $>0$ Then

"If find the corresponding Chinese name in the library'

Data1.Recordset.movefirst

richtextbox1.loadfile Str \& txtname.Text

" Call the corresponding text information'

image1.Picture = LoadPicture(Str \& "datalpicturel" \& Data1.Recordset.Fields ("picture" \& m)". Value)

"Call the corresponding picture information'

End If

latin.Text $=$ Data1.Recordset.Fields ("English name").Value

"display English name corresponding with Chinese name '

End If

End Sub

'"End'

We can get more information about a disease when we know the disease name. Click button of "query according to the disease name" in the query interface shown in Fig. 4. Select the name of the disease in the list, and then click the "search" button. It came to the interface of query result (Fig. 5).

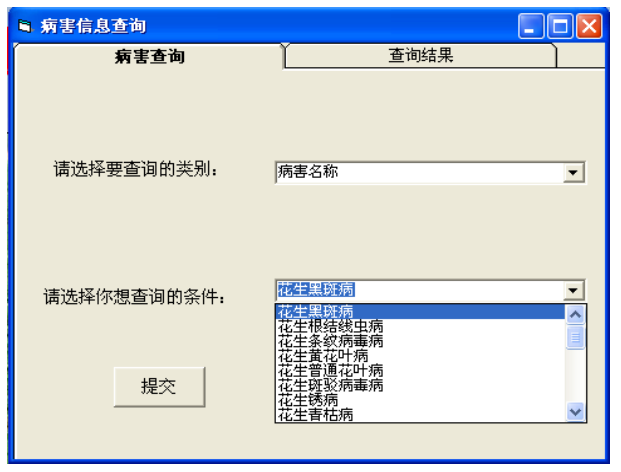

Fig. 4. Disease query interface

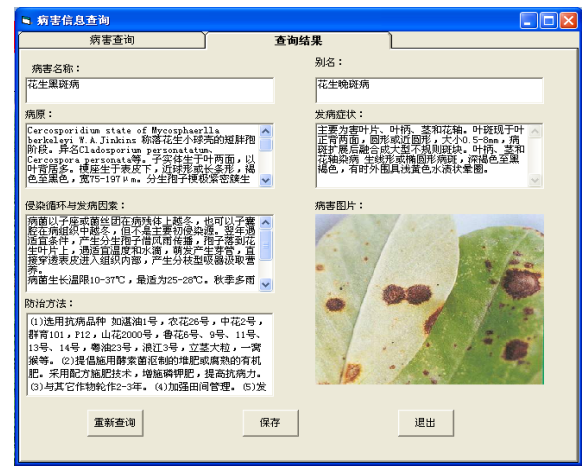

Fig. 5. Disease query result interface

\section{Conclusion}

The system adopts the modular design, which only needs to improve and expand the specific modules. It can effectively provide the details of peanut diseases and the 
comprehensive control technical point of diseases, through collecting the massive peanut disease relevant knowledge, and analysising comprehensively the knowledge base. The system can provide perfect consult service for agricultural technicians and farmers against peanut diseases, change the present situation of blind use of pesticides, and protect the ecological environment. So it can play an important role, and also can alleviate the situation of seriously lack of the advanced agricultural technology personnel. So it has practical significance to promote peanut diseases diagnosis and prevention expert system. The accuracy of the model and fitting in with the actual production are the critical factors to decide the success of the system, therefore it also need continuous improvement to verify the system in future.

Acknowledgment. The project received financial support from the China Agriculture Research System (CARS-14).

\section{References}

1. Wan, S.: Peanut cultivation in China. Shanghai Science and Technology Press, Shanghai (2003)

2. Wang, Z., Gai, S.: Shandong Peanut. Shanghai Science and Technology Press, Shanghai (1999)

3. Sun, H., Ding, J., Li, Y.: Expert system (ES) and its application in agriculture. Journal of Shandong Agricultural University 29(2), 270-276 (1998)

4. Ghoshi, S.: Teapest: An expert system for insect pest management in tea. Applied Engineering in Agriculture 19(5), 619-625 (2003)

5. Liu, D., Zhuang, T.: Maize diseases and pests control expert system. Research and Development of Computer 36(1), 36-41 (1999)

6. Zheng, Y., Cheng, J., Zhang, Q.: A preliminary study on the main diseases and insect pests of vegetables consulting system in Zhejiang Province. Zhejiang Journal of Agricultural Sciences 16(4), 186-191 (2004)

7. Shao, G., Li, Z., Wang, W., et al.: Study on VPRDES of vegetable diseases remote diagnosis expert system in Beijing area. Plant Protection 32(1), 51-54 (2006)

8. Ma, Q., Kong, J.: Utility color atlas on economic crops diseases and insect pests. Henan Science and Technology Press, Zhengzhou (1998)

9. Tu, Y.: Pesticide application technology standardization. China Standard Press, Beijing (2001)

10. Niu, Z., Yang, X., Shou, S., et al.: Research and design of cucumber disease diagnosis expert system knowledge organization. System Sciences and Comprehensive Studies 20(1), 33-36 (2004)

11. Lian, S.: Introduction to artificial intelligence technology, pp. 34-42. Xi'an Electronic and Science University Press, Xi' an (2001)

12. Giarratano, J., Riley, G.: Principles and programming on expert systems, pp. 67-75. Machinery Industry Press, Beijing (2000)

13. Black, R., Sweetmore, A., Holt, J.: Plant clinic: a training system for decision-making and resource management in plant disease diagnosis. 4, 52-55 (1995) 